\title{
Lapatinib in combination with capecitabine versus continued use of trastuzumab in breast cancer patients with trastuzumab-resistance: a retrospective study for Chinese population
}

fan Yang

First Affiliated Hospital of nanjing Medical University

\section{Xiang Huang}

Nanjing Medical University first affiliated hospital

Chunxiao Sun

the first affiliated hospital of nanjing medical university

Jianbin Li

Chinese People's Liberation Army Hospital 307

\section{Biyun Wang}

Fudan University Shanghai Cancer Center

\section{Min Yan}

Henan Cancer Hospital

Feng Jin

the first afficialited hospital of china medical university

Haibo Wang

Qingdao University Medical College

Jin Zhang

Tianjin Agricultural University cancer institution and hospital

\section{Peifen Fu}

Zhejiang University

\section{Tianyu Zeng}

First Affiliated Hospital of nanjing Medical University

Jian Wang

First Affiliated Hospital of nanjing Medical University

Wei Li

First Affiliated Hospital of nanjing Medical University

\section{Yongfei Li}

First Affiliated Hospital of nanjing Medical University

\section{Mengzhu Yang}

First Affiliated Hospital of nanjing Medical University 


\section{Jun Li}

First Affiliated Hospital of nanjing Medical University

\section{Hao Wu}

First Affiliated Hospital of nanjing Medical University

\section{Ziyi Fu}

nanjing medical university

\section{Yongmei Yin ( $\sim$ ymyin@njmu.edu.cn )}

the First Affiliated Hospital of Nanjing Medical University https://orcid.org/0000-0003-3335-369X zefei jiang

Chinese People's Liberation Army Hospital 307

\section{Research article}

Keywords: Lapatinib; Trastuzumab; Resistance; Breast cancer

Posted Date: December 30th, 2019

DOI: https://doi.org/10.21203/rs.2.16713/v2

License: (c) (i) This work is licensed under a Creative Commons Attribution 4.0 International License. Read Full License 


\section{Abstract}

Background: The efficacy and safety of lapatinib plus capecitabine (LC or LX) versus trastuzumab plus chemotherapy in patients with HER-positive metastatic breast cancer who were resistant to trastuzumab is largely unknown. Methods: we retrospectively analyzed breast cancer patients who began the treatment with regimen of lapatinib plus capecitabine (LC or LX) or trastuzumab beyond progression (TBP) at eight hospitals between May 2010 and October 2017. Results: Among 554 patients who had developed resistance to trastuzumab, the median PFS was 6.77 months in the LX group compared with 5.6 months in the TBP group (hazard ratio $0.804 ; 95 \% \mathrm{Cl}, 0.67$ to $0.96 ; \mathrm{P}=0.019$ ). The central nervous system progression rate during treatment was $5.9 \%$ in LX group and $12.5 \%$ in TBP group, respectively $(P=0.018)$. Conclusion: The combination of lapatinib and capecitabine has shown a prolonged PFS compared with TBP in patients who had progressed on trastuzumab.

\section{Background}

Breast cancer is one of the most common invasive cancers and expected to account for $14 \%$ of all cancer deaths in women world-wide[1]. Activation and over-expression of epidermal growth factor receptor (EGFR, also known as ErbB) family members, including EGFR (ErbB1 or HER1), HER3 (ErbB3), HER4 (ErbB4), and HER2 (ErbB2), govern multiple important cellular processes in breast cancer.

Activation of HER2, a tyrosine kinase receptor, induces homo- and hetero-dimerization, which leads to the activation of downstream effectors and pathways such as PI3K/AKT and RAS/MAPK[2]. Amplification of the HER2 gene and/or over-expression of its protein product occurs in approximately $20-25 \%$ breast cancers[3]. Clinically, HER2-positive tumors are characterized by aggressive clinical course and poor overall prognosis[4]. The introduction of the anti-HER2 monoclonal antibody trastuzumab has dramatically improved the poor prognosis of this population of patients[5-7]. Trastuzumab binds to the extracellular domain of the HER2 receptor, prevents receptor homo- and hetero-dimerization, therefore inhibiting the activation of downstream oncogenic signaling[8]. Trastuzumab-containing treatment is the standard approach for HER-2 positive metastatic breast cancer. Despite its overall clinical efficacy, de novo and acquired resistance to trastuzumab administration have been observed[9]. The development of distant metastasis to liver, bone, lung and brain has become a major challenge in management of patients with HER-2 positive breast cancer, probably due to longer life expectancy and acquired trastuzumab resistance[10]. Therefore, it is urgently needed to develop new strategy of salvage therapy for patients who have developed resistance to trastuzumab.

However, the consensus guidelines on targeted treatment for resistance in HER2-positive breast cancer are not available[11,12]. Combination of anti-HER2 agents with chemotherapy, anti-HER2/HER3 dimerization, or inhibitors of the downstream signaling might improve prognosis[13]. Fujimoto-Ouchi demonstrated that trastuzumab in combination with taxanes or capecitabine showed antitumor activity in trastuzumab resistant model[14]. The GBG 26/BIG 3-05 enrolled patients with HER2-positive metastatic breast cancer (Stage IV) that progresses during treatment with trastuzumab. Among these patients, 78 patients were randomly assigned to receive capecitabine, and 78 patients to capecitabine plus 
trastuzumab. The results showed that median TTP were 5.6 months vs 8.2 months, $P=0.0338[15]$. In a similar study, patients who received trastuzumab treatment beyond progression (TBP) had longer median OS than those who terminated (21.3 months VS 4.6 months (P凶0.0001))[16]. Taken together, these studies suggest that a clinical benefit has been observed for treatment with trastuzumab beyond progression.

Lapatinib, an orally active, small molecule, tyrosine kinase inhibitor, has shown non-cross resistance with trastuzumab. It binds reversibly to the cytoplasmic domains of both EGFR and HER2 that in turn blocks the activating signaling cascades in the MAPK and PI3K pathways[17]. Given its unique mechanistic function, lapatinib might be a suitable option in HER2-positive MBC that have become resistant to suppression by trastuzumab. Studies have also shown that the phosphorylation of p95 HER2 (a truncated version lacking the extracellular domain) and the formation of heterodimers between HER2 and other members of the HER family might be inhibited by lapatinib, but not trastuzumab[18, 19]. In EGF100151 trial, lapatinib plus capecitabine reduced the hazard for time-to-disease progression (hazard ratio $0.49 ; 95 \% \mathrm{Cl} 0.34-0.71 ; P<0.001$ ) in HER2-positive breast cancer that progressed on anthracycline, a taxane and trastuzumab[11, 20]. In 2010, the US FDA approved the use of lapatinib in combination with capecitabine for the treatment of patients with HER2-positive MBC. In addition, lapatinib in combination with capecitabine shows excellent activity against central nervous system (CNS) metastases. An study suggested that patients with brain metastasis achieved significant longer overall survival in the lapatinib group compared with trastuzumab-based therapy (19.1 vs. 12 months, $P=0.039)$ [21].

Clinical trials have demonstrated that other HER-2 targeted agents, such as T-DM1 and pertuzumab, have shown efficacy in patients pretreated with trastuzumab[22, 23]. However, these regimens remain unavailable in China. Therefore, trastuzumab plus chemotherapy or switching to the lapatinib plus capecitabine regimen are common options for most Chinese patients who have developed resistance to trastuzumab. No compelling evidence indicates if certain patients benefit more from continuation of trastuzumab compared with switching to lapatinib. In the present analysis, we compare the clinical outcome of continuing trastuzumab treatment or replacing trastuzumab with lapatinib for metastatic breast cancer $(\mathrm{MBC})$ patients who are resistant to trastuzumab.

\section{Materials And Methods}

\section{Patients}

We retrospectively reviewed the medical records of HER2-positive metastatic breast cancer patients at eight hospitals from May 2010 to October 2017. HER-2 status was considered positive if immunohistochemistry (IHC) test showing +++ or if HER2 gene amplification was found by fluorescence in situ hybridization. Female patients who received lapatinib plus capecitabine or trastuzumab plus chemotherapy after developing resistance to trastuzumab were included. Primary resistance was defined as new recurrences diagnosed on or within 12 months after the end of (neo)adjuvant trastuzumab or progressed at first radiological reassessment at 8-12 weeks or within 3 months at trastuzumab therapy 
for metastatic disease. Secondary resistance was defined as disease progression of metastatic cancer occurring on trastuzumab-containing regimens that initially achieved disease response or stabilization at first radiological assessment. We exclude patients whose therapeutic regimen has been administered beyond the third line for recurrent metastatic breast cancer and those received anti HER2 therapies except for trastuzumab. Patients have central nervous system metastasis were previously treated with radiotherapy or surgery. All patients who have at least one measurable disease and tumor response were evaluated according to Response Evaluation Criteria in Solid Tumors criteria 1.1..

\section{Endpoint}

The primary endpoint was PFS, defined as time from the initiation of TBP or LX until the earliest date of disease progression or death. Secondary included ORR (the ratio of patients who have complete or partial tumor remission), CBR (clinical benefit rate), defined as the ratio of patients who have complete or partial tumor remission or stable disease for more than 6 months.

\section{Statistical analysis}

Statistical analyses were performed using SPSS version 24.0 (SPSS Inc, Chicago, IL, USA). A two-tailed $P<0.05$. Kaplan-Meier estimate was used to compare PFS using the log-rank test. Comparisons of ORR, $\mathrm{CBR}$, Central nervous system progression rate was conducted using chi-square test. Categorical variables were compared between the groups by chi-square tests too. The effect of various baseline covariates on PFS was analyzed by Cox regression modeling.

\section{Results}

\section{Patient characteristics}

A total of 554 patents were identified and the median follow-up time was 15 months. The demographic characteristics between the two groups were showed in Table 1 and the most variables were wellbalanced. A higher proportion of patients in the TBP group was older than 50 years and had HR-positive tumors. 94 (36.9\%) patients received lapatinib plus capecitabine (LX) and 164 (54.8\%) patients received trastuzumab beyond progression (TBP) in the second line treatment $(P<0.001)$. While in the third line treatment, 124 (48.6\%) patients received lapatinib plus capecitabine (LX) and $92(30.8 \%)$ patients received trastuzumab beyond progression (TBP) $(P=0.001)$, which indicated more patients received $L X$ in later lines. The predominant chemotherapy combined with trastuzumab was taxane (Table 2).

\section{Efficacy}

The median PFS was 6.77 months in the LX group compared with 5.6 months in the TBP group (hazard ratio $0.7955 ; 95 \% \mathrm{Cl}, 0.6632$ to 0.9542 ; log-rank $P=0.014$; Figure $1 \mathrm{~A})$. In the primary resistant patients, the median was significantly increased from 4.3 months for TBP to 6.8 months for $L X(P<0.001$; Figure 1B). In the secondary resistant patients, no significant difference was observed (median PFS: 6.6 months for LX VS 6.3 months for TBP, $P=0.8827$; Figure $1 \mathrm{C}$ ). The best overall response to treatment was not 
evaluable in 64 patients. We observed no significant difference in the ORR and CBR between two groups $(P=0.822 ; P=0.224 ;$ eTable 1 in Supplement 1$)$.

\section{First line treatment}

In the TBP group, 3 (7\%) patients progressed on (neo)adjuvant trastuzumab therapy and $40(93 \%)$ patients progressed within 12 months after completing (neo)adjuvant therapy. In the LX group, 3 (8.2\%) patients relapsed on and 34 (91.8\%) patients relapsed within 12 months after the end of (neo)adjuvant trastuzumab treatment. Hence, they are all primary resistant to trastuzumab. The median PFS was 7.9 months in the LX group compared with 4.4 months in the TBP group (hazard ratio $0.4565 ; 95 \% \mathrm{Cl}, 0.2754$ to 0.7566 ; log-rank $P=0.002$; Figure 2). 15 patients were not evaluable in best response to treatment. The ORR was significantly increased from $8.3 \%$ for TBP to $27.6 \%$ for $\operatorname{LX}(P=0.04)$. The CBR was significantly improved too (36.1\% to $69 \%, P=0.008$; eTable 2 in Supplement 1$)$.

\section{Second and third line treatment}

After developing resistance to the trastuzumab-containing treatment, 218 patients received LX, and 256 patients continued using trastuzumab in the later lines. The result showed that median PFS was 6.6 months for LX group compared with 5.9 months for TBP group (hazard ratio $0.8605 ; 95 \% \mathrm{Cl}, 0.7068$ to 1.048; log-rank $P=0.135$; Figure $3 \mathrm{~A}$ ). No improvement in median PFS was observed. Median PFS in the primary resistant population increased from 4.3 months for TBP to 6.6 months for LX group (hazard ratio $0.5057 ; 95 \% \mathrm{Cl}, 0.335$ to 0.7633 ; log-rank $P=0.001$; Figure $3 \mathrm{~B}$ ). The best response to treatment was missing in 22 patients in the second line setting. The ORR and CBR between the two groups have no statistical difference (eTable 3 in Supplement 1). In the third line setting, 27 patients were not evaluable in best response to treatment. We found no statistical difference in ORR or CBR (eTable 4 in Supplement 1).

\section{Multivariate analysis}

We carried out a multivariate analysis to investigate whether the anti-HER2 therapy effect was different according to baseline characters. The model included treatment after resistance to trastuzumab, age, hormone receptor status, metastatic sites, treatment line. We noted that secondary or primary resistance had a differential prognostic effect in trastuzumab treated patients, the HR for PFS favoured patients who were secondary resistance (Figure 4).

\section{Central nervous system metastases}

Response in the CNS was evaluable in 451 patients. 58 patients have baseline central nervous system metastases, all received prior local therapy and presented in Table3. 3 patients in the LX group and 4 patients in the TBP group have more than 3 metastatic sites in the brain. In the patients with baseline CNS metastases, we observed 6 progressive disease in the LX group. While in the TBP group, patients who showed progression were 20. On the patients without baseline CNS metastases, $2.96 \%$ (6/203) and $4.44 \%(11 / 248)$ developed new CNS metastases in the LX and TBP group during the treatment. CNS progression rate was $5.9 \%$ and $12.5 \%$ respectively ( $P=0.018$; Table 4$)$. 


\section{Safety}

The most common adverse events were neutropenia, thrombocytopenia and hand-foot syndrome. 42 $(17.8 \%)$ patients in the LX group and $61(20.6 \%)$ patients in the TBP group experienced grade 3 or 4 toxicities $(P=0.415)$. The most frequent grade III-IV AEs were diarrhoea $(5.1 \%)$, hand-foot syndrome (10.2\%) in LX group, and ALT/AST increased (9.1\%) and neutropenia (6.4\%) in TBP group. Treatmentrelated LVEF decline was observed in 2 patients in the trastuzumab group but was moderate in severity (Table 5). This study was retrospective by nature, the adverse events may be underestimated.

\section{Discussion}

Our study provided evidence that if patients were resistant to trastuzumab, switching to the combination of lapatinib and capecitabine had longer PFS than those patients continued use of trastuzumab. Findings from our analysis suggest that the effect of lapatinib on PFS may be explained by excellent effect in primary resistant patients.

The results of the current study accord with two small randomized trials comparing capecitabine plus lapatinib with trastuzumab plus lapatinib as treatment for patients progressed on trastuzumabcontaining therapy. An analysis of 86 women who were HER-2 positive, locally advanced breast cancer or metastatic breast cancer (MBC) and developed resistance to trastuzumab demonstrated that the trastuzumab combined with capecitabine led to a not significant inferior PFS compared with lapatinib ,

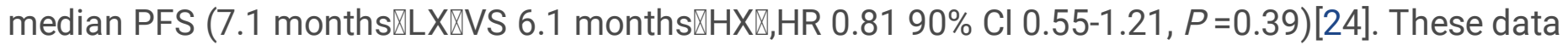
were supported by study results from Bian, et al $₫$ who randomly assigned $120 \mathrm{HER}-2$ positive MBC patients with resistance to trastuzumab in a 1:1 ratio to receive capecitabine with either trastuzumab or lapatinib, median PFS (4.5 months VS 6 months, $\mathrm{HR}=0.61 \otimes 95 \% \mathrm{Cl} \otimes 0.42-0.88 \otimes P=0.006)[25] .30 \%$ of patients in trastuzumab group and $55 \%$ in the lapatinib group experienced a PFS longer than 6 months. In consistence with those reports, our study suggests that patients can respond to further HER2-directed regimens after development of resistance to HER2- directed therapy. The optimal anti-HER2 treatment for patients who failed trastuzumab treatment in clinical practice would be lapatinib when pertuzumab / TDM1 are not available.

Our findings differ in part from two studies that compare tyrosine kinase inhibitors with trastuzumab for HER2-overexpressing metastatic breast cancer. In the LUX-Breast 1 trial[26], an oral irreversible ErbB family blocker afatinib plus vinorelbine resulted in similar PFS compared with trastuzumab plus vinorelbine in HER2-positive metastatic breast cancer who had progressed on trastuzumab. Median PFS was 5.5 months in the afatinib group and 5.6 months in the trastuzumab group (hazard ratio $1.1095 \% \mathrm{Cl}$ $0.86-1.41 ; P=0.43)$. For patients receiving first-line therapy, PFS was also insignificantly different between the afatinib and trastuzumab-based therapy (hazard ratio $1.10295 \% \mathrm{Cl} 0.759-1.600 ; P=0.61$ ). In the MA.31 trial, PFS was shorter for lapatinib plus taxane compared with trastuzumab plus taxane administrated as first-line therapy of metastatic breast cancer (9.0 months VS 11.3 months; HR 1.37 [95\% $\mathrm{Cl} 1.13-1.65] ; P=0.001$ )[27]. The trial was terminated earlier. However, although afatinib is a second- 
generation, broader inhibitor of the ErbB family of proteins[28], no randomized trials to compare the efficacy of afatinib with lapatinib in women progressed during trastuzumab treatment. Furthermore, a major difference between MA.31 trial and our study was that a large part of patients were newly diagnosed advanced breast cancer and were trastuzumab-naïve. This might affect the survival outcome.

Lapatinib has a different mechanism of inhibition on HER2 and EGFR signaling compared with trastuzumab. Preclinical evidence suggests non-cross resistance of trastuzumab and lapatinib. PTEN abrogates phosphatidyl inositol-3-kinase (PI3K), which results in inhibition of Akt signaling. Non-existent or limited expression of PTEN (phosphatase and tensin homologue deleted on chromosome 10), might be a marker of resistance to trastuzumab[29]. Previous studies have confirmed PTEN expression has no correlation with response to lapatinib[30]. IGF-1R (insulin-like growth factor receptor) is important for cell proliferation and survival[31]. It has been reported that overexpression of IGF-1R predicted resistance to trastuzumab in breast cancer cells[31-33]. IGF-1R belongs to the tyrosine kinase receptor family and breast cancer cells that express IGF-1R may still be sensitive to lapatinib[34].

We try to identify subsets of patients who would derive the greatest benefit from further HER2-directed therapy. To this end, we examined whether the prognosis in the primary resistant patients paralleled those of the secondary resistant to HER2-directed therapy. Indeed, in multiple lines, the data showed that those primary resistant patients who received LX tended to have longer PFS with statistical significance, while the PFS of secondary resistant patients receiving the TBP regimen was similar with the patients receiving the LX regimen. p95 HER2 (a truncated version lacking the extracellular domain) would preventtrastuzumab binding and are associated with poor prognosis in patients. Lapatinib inhibits p95HER2 phosphorylation while trastuzumab doesn't[35]. That may explain why switching to lapatinib predicts for extended PFS in the primary resistant group.

Unlike primary resistant patients, a clinical benefit was observed for treatment with trastuzumabcontaining regimen in patients acquired resistance to anti-HER-2 therapy. Trastuzumab might have additional anti-tumour efficacy via antibody-dependent cellular-cytotoxicity (ADCC), by which induces immune effector cells to kill cancer cells[36, 37].

We also found patients in the second line treatment had a higher proportion of trastuzumab beyond progression therapy than those in the third-line setting. The predominant HER-2 targeted therapy in the second line setting was trastuzumab instead of lapatinib. A plausible reason for the disparities concerns the assumption that patient refractory to prior chemotherapy agent but not to trastuzumab itself. Secondly, anti-HER2 therapy is expensive and time consuming, and varying medical insurance policies may contribute to the continued use of trastuzumab.

Breast cancer patients with HER2 overexpression have greater risk for developing brain metastasis, and trastuzumab treatment has emerged as a factor contributing to this risk[38]. Previous studies support the hypothesis that brain is a 'sanctuary' site for the development of metastases due to the limited ability of trastuzumab in penetrating the Blood Brain Barrier (BBB)[39]. Lapatinib is a small dual tyrosine-kinase inhibitor of HER1 and HER2 with a hypothetical ability to cross the BBB[40]. The combination of lapatinib 
with capecitabine has central nervous system (CNS) activity for the treatment of patients with HER2positive brain metastatic breast cancer. Clinical evidence supports that patients with HER2-positive brain metastasis achieved a significant clinical benefit from lapatinib and capecitabine both as single agents and in combination in patients with brain metastases[41-43]. In the present study, the percentage of patients with central nervous system progression was higher in the TBP group. In addition, the comparison of CNS progression rate indicates that lapatinib is more effective in brain metastases than trastuzumab. These findings are consistent with the result of a randomized clinical trial which evaluate the effect of neratinib compared with trastuzumab in previously untreated metastatic ERBB2-Positive Breast Cancer. Neratinib, another kind of oral irreversible ERBB family blocker, was associated with fewer central nervous system recurrences (relative risk, $0.48 ; 95 \% \mathrm{Cl}, 0.29-0.79 ; P=0.002$ ) and also delayed the time to CNS relapses compared with trastuzumab (HR, 0.45; 95\% Cl, 0.26-0.78; $P=0.004)$ [44]. In EMILIA trial, modest activity of lapatinib plus capecitabine upon CNS recurrences, 2.0\% (9/450) in the T-DM1 group and $0.7 \%(3 / 446)$ in the LX group developed new brain metastases[22, 45]. It appears that switching patients with treated brain metastases to lapatinib-containing treatment more effectively prevent brain progression.

It should be noted that there were a few limitations in our study. First, it's retrospectively, potential imbalance in contributing factors of patient prognosis and patient heterogeneity in terms of treatment does exist. For example, women who switching to lapatinib were younger and more likely to achieve antitumor activity of new anti-HER2 regimen. Second, patients who received chemotherapy and trastuzumab sequentially or concomitantly may affect the prognosis. Third, some data could not be abstracted from medical records and missing.

\section{Conclusions}

In conclusion, these data confirm that after developing resistance to trastuzumab, patients can still derive benefit from HER-2 targeted therapy. The combination of lapatinib and capecitabine has shown a prolonged survival compared with TBP in patients with prior trastuzumab exposure.

\section{List Of Abbreviations}

HER2 human epidermal growth factor receptor 2

LC or LX lapatinib plus capecitabine

TBP trastuzumab beyond progression

PFS progression-free survival

OS overall survival

RECIST Response Evaluation Criteria In Solid Tumors 
CTCAE Common Terminology Criteria for Adverse Events

ORR objective response rate

DCR disease control rate

HR hazard ratio

$\mathrm{Cl}$ confidence interval

ADCC antibody-dependent cell-mediated cytotoxiciy

NCCN National Comprehensive Cancer Network

EGFR epidermal growth factor receptor

FDA Food and Drug Administration

ECOG Eastern Cooperative Oncology Group

CR complete response

PR partial response

PD progressive disease

SD stable disease

PI3K phosphatidyl inositol 3-kinase

IGF-1R insulin-like growth factor-1 receptor

mTOR mammalian target of rapamycin

SRS stereotactic radiosurgery

WBRT whole brain radiotherapy;

$\mathrm{NCI}$ CTC National Cancer Institute Common Terminology Criteria

\section{Declarations}

\section{Ethics approval and consent to participate}

The study was approved by First Affiliated Hospital of Nanjing Medical University (Nanjing, China) and written informed consent from each patient was obtained. The use of samples was approved by the Ethics Committee of the First Affiliated Hospital of Nanjing Medical University. 


\section{Consent for publication}

Written informed consent for publication of their clinical details was obtained from the patients.

\section{Availability of data and material}

The datasets during and analysed during the current study available from the corresponding author on reasonable request.

\section{Competing interests}

The authors declare that they have no competing interests.

\section{Funding}

This study was financially supported by the collaborative innovation center for tumor individualization focuses on open topics, Grant/Award Number: JX21817902/008; 333 Project of Jiangsu Province, Grant/Award Numbers: BRA2015470, BRA2017534; High-level innovation team of Nanjing Medical University, Grant/Award Number: JX102GSP201727; Project of China key research and development program precision medicine research, Grant/Award Number: 2016YFC0905901; Key medical talents, Grant/Award Number: ZDRCA2016023; National Key Research and Development Program of China, Grant/Award Number: ZDZX2017ZL-01; Wu Jieping Foundation, Grant/Award Number: 320.6750.17006.

\section{Authors' contribution}

Conception/design: Yongmei Yin

Provision of study material or patients: Zefei Jiang, Yongmei Yin, Biyun Wang, Min Yan, Feng Jin , Yongmei Yin, Haibo Wang, Jin Zhang, Peifen Fu

Collection and/or assembly of data: Fan Yang, Tianyu Zeng, Xiang Huang, Chunxiao Sun, Mengzhu Yang, Jianbin Li, Zefei Jiang, Yongmei Yin, Biyun Wang, Min Yan, Feng Jin , Yongmei Yin, Haibo Wang, Jin Zhang, Peifen Fu

Data analysis and interpretation:Fan Yang, Xiang Huang, Tianyu Zeng

Manuscriptwriting:Fan Yang

Final approval of manuscript: Yongmei Yin

Acknowledgements

Not applicable

\section{References}


1. Siegel RL, Miller KD, Jemal A. Cancer Statistics, 2017. CA Cancer J Clin 2017; 67: 7-30.

2. Rubin I, Yarden Y. The basic biology of HER2. Ann Oncol 2001; 12 Suppl 1: S3-8.

3. Wolff AC, Hammond ME, Hicks DG et al. Recommendations for human epidermal growth factor receptor 2 testing in breast cancer: American Society of Clinical Oncology/College of American Pathologists clinical practice guideline update. Arch Pathol Lab Med 2014; 138: 241-256.

4. Ross JS, Slodkowska EA, Symmans WF et al. The HER-2 receptor and breast cancer: ten years of targeted anti-HER-2 therapy and personalized medicine. Oncologist 2009; 14: 320-368.

5. Romond EH, Perez EA, Bryant J et al. Trastuzumab plus adjuvant chemotherapy for operable HER2positive breast cancer. N Engl J Med 2005; 353: 1673-1684.

6. Marty M, Cognetti F, Maraninchi D et al. Randomized phase II trial of the efficacy and safety of trastuzumab combined with docetaxel in patients with human epidermal growth factor receptor 2positive metastatic breast cancer administered as first-line treatment: the M77001 study group. J Clin Oncol 2005; 23: 4265-4274.

7. Cameron D, Piccart-Gebhart MJ, Gelber RD et al. 11 years' follow-up of trastuzumab after adjuvant chemotherapy in HER2-positive early breast cancer: final analysis of the HERceptin Adjuvant (HERA) trial. Lancet 2017; 389: 1195-1205.

8. Hudis CA. Drug therapy: Trastuzumab - Mechanism of action and use in clinical practice. New England Journal of Medicine 2007; 357: 39-51.

9. Mohd Sharial MS, Crown J, Hennessy BT. Overcoming resistance and restoring sensitivity to HER2targeted therapies in breast cancer. Ann Oncol 2012; 23: 3007-3016.

10. Gajria D, Chandarlapaty S. HER2-amplified breast cancer: mechanisms of trastuzumab resistance and novel targeted therapies. Expert Review of Anticancer Therapy 2011; 11: 263-275.

11. Geyer CE, Forster J, Lindquist D et al. Lapatinib plus capecitabine for HER2-positive advanced breast cancer. N Engl J Med 2006; 355: 2733-2743.

12. Harbeck N, Gnant M. Breast cancer. Lancet 2017; 389: 1134-1150.

13. Wong $\mathrm{H}$, Leung $\mathrm{R}$, Kwong A et al. Integrating Molecular Mechanisms and Clinical Evidence in the Management of Trastuzumab Resistant or Refractory HER-2(+) Metastatic Breast Cancer. Oncologist 2011; 16: 1535-1546.

14. Fujimoto-Ouchi K, Sekiguchi F, Yamamoto K et al. Preclinical study of prolonged administration of trastuzumab as combination therapy after disease progression during trastuzumab monotherapy. Cancer Chemotherapy and Pharmacology 2010; 66: 269-276.

15. von Minckwitz G, du Bois A, Schmidt M et al. Trastuzumab Beyond Progression in Human Epidermal Growth Factor Receptor 2-Positive Advanced Breast Cancer: A German Breast Group 26/Breast International Group 03-05 Study. Journal of Clinical Oncology 2009; 27: 1999-2006.

16. Hammerman A, Greenberg-Dotan S, Feldhamer I et al. Second-Line Treatment of Her2-Positive Metastatic Breast Cancer: Trastuzumab beyond Progression or Lapatinib? A Population Based Cohort Study. PLoS One 2015; 10: e0138229. 
17. Mukherjee A, Dhadda AS, Shehata M, Chan S. Lapatinib: a tyrosine kinase inhibitor with a clinical role in breast cancer. Expert Opinion on Pharmacotherapy 2007; 8: 2189-2204.

18. Nishimura R, Toh U, Tanaka M et al. Role of HER2-Related Biomarkers (HER2, p95HER2, HER3, PTEN, and PIK3CA) in the Efficacy of Lapatinib plus Capecitabine in HER2-Positive Advanced Breast Cancer Refractory to Trastuzumab. Oncology 2017; 93: 51-61.

19. Tural D, Akar E, Mutlu H, Kilickap S. P95 HER2 fragments and breast cancer outcome. Expert Review of Anticancer Therapy 2014; 14: 1089-1096.

20. Cameron D, Casey M, Oliva C et al. Lapatinib plus capecitabine in women with HER-2-positive advanced breast cancer: final survival analysis of a phase III randomized trial. Oncologist 2010; 15 : 924-934.

21. Kaplan MA, Isikdogan A, Koca D et al. Clinical outcomes in patients who received lapatinib plus capecitabine combination therapy for HER2-positive breast cancer with brain metastasis and a comparison of survival with those who received trastuzumab-based therapy: a study by the Anatolian Society of Medical Oncology. Breast Cancer 2014; 21: 677-683.

22. Diéras $V$, Miles $D$, Verma $S$ et al. Trastuzumab emtansine versus capecitabine plus lapatinib in patients with previously treated HER2-positive advanced breast cancer (EMILIA): a descriptive analysis of final overall survival results from a randomised, open-label, phase 3 trial. The Lancet Oncology 2017; 18: 732-742.

23. Baselga J, Cortes J, Kim SB et al. Pertuzumab plus Trastuzumab plus Docetaxel for Metastatic Breast Cancer. New England Journal of Medicine 2012; 366: 109-119.

24. Takano T, Tsurutani J, Takahashi $\mathrm{M}$ et al. A randomized phase II trial of trastuzumab plus capecitabine versus lapatinib plus capecitabine in patients with HER2-positive metastatic breast cancer previously treated with trastuzumab and taxanes: WJOG6110B/ELTOP. Breast 2018; 40: 6775.

25. Bian L, Wang T, Zhang S, Jiang Z. Trastuzumab plus capecitabine vs. lapatinib plus capecitabine in patients with trastuzumab resistance and taxane-pretreated metastatic breast cancer. Tumour Biol 2013; 34: 3153-3158.

26. Harbeck N, Huang C-S, Hurvitz $S$ et al. Afatinib plus vinorelbine versus trastuzumab plus vinorelbine in patients with HER2-overexpressing metastatic breast cancer who had progressed on one previous trastuzumab treatment (LUX-Breast 1): an open-label, randomised, phase 3 trial. The Lancet Oncology 2016; 17: 357-366.

27. Pivot X, Manikhas A, Zurawski B et al. CEREBEL (EGF111438): A Phase III, Randomized, Open-Label Study of Lapatinib Plus Capecitabine Versus Trastuzumab Plus Capecitabine in Patients With Human Epidermal Growth Factor Receptor 2-Positive Metastatic Breast Cancer. J Clin Oncol 2015; 33: 1564-1573.

28. Solca F, Dahl G, Zoephel A et al. Target Binding Properties and Cellular Activity of Afatinib (BIBW 2992), an Irreversible ErbB Family Blocker. Journal of Pharmacology and Experimental Therapeutics 2012; 343: 342-350. 
29. Nagata Y, Lan KH, Zhou XY et al. PTEN activation contributes to tumor inhibition by trastuzumab, and loss of PTEN predicts trastuzumab resistance in patients. Cancer Cell 2004; 6: 117-127.

30. Xu B, Guan Z, Shen Z et al. Association of phosphatase and tensin homolog low and phosphatidylinositol 3-kinase catalytic subunit alpha gene mutations on outcome in human epidermal growth factor receptor 2-positive metastatic breast cancer patients treated with first-line lapatinib plus paclitaxel or paclitaxel alone. Breast Cancer Res 2014; 16: 405.

31. Nahta R, Yuan LYH, Zhang B et al. Insulin-like growth factor-I receptor/human epidermal growth factor receptor 2 heterodimerization contributes to trastuzumab resistance of breast cancer cells. Cancer Research 2005; 65: 11118-11128.

32. Gallardo A, Lerma E, Escuin D et al. Increased signalling of EGFR and IGF1R, and deregulation of $\mathrm{PTEN} / \mathrm{PI}$ 3K/Akt pathway are related with trastuzumab resistance in HER2 breast carcinomas. $\mathrm{Br} \mathrm{J}$ Cancer 2012; 106: 1367-1373.

33. Sanabria-Figueroa E, Donnelly SM, Foy KC et al. Insulin-like growth factor-1 receptor signaling increases the invasive potential of human epidermal growth factor receptor 2-overexpressing breast cancer cells via Src-focal adhesion kinase and forkhead box protein M1. Mol Pharmacol 2015; 87: 150-161.

34. Saxena NK, Taliaferro-Smith L, Knight BB et al. Bidirectional crosstalk between leptin and insulin-like growth factor-I signaling promotes invasion and migration of breast cancer cells via transactivation of epidermal growth factor receptor. Cancer Res 2008; 68: 9712-9722.

35. Scaltriti M, Rojo F, Ocana A et al. Expression of p95HER2, a truncated form of the HER2 receptor, and response to anti-HER2 therapies in breast cancer. Journal of the National Cancer Institute 2007; 99 : 628-638.

36. Bianchini G, Gianni L. The immune system and response to HER2-targeted treatment in breast cancer. Lancet Oncol 2014; 15: e58-68.

37. Park S, Jiang ZJ, Mortenson ED et al. The Therapeutic Effect of Anti-HER2/neu Antibody Depends on Both Innate and Adaptive Immunity. Cancer Cell 2010; 18: 160-170.

38. Leyland-Jones B. Human Epidermal Growth Factor Receptor 2-Positive Breast Cancer and Central Nervous System Metastases. Journal of Clinical Oncology 2009; 27: 5278-5286.

39. Arslan UY, Oksuzoglu B, Aksoy S et al. Breast cancer subtypes and outcomes of central nervous system metastases. Breast 2011; 20: 562-567.

40. Sutherland S, Ashley S, Miles D et al. Treatment of HER2-positive metastatic breast cancer with lapatinib and capecitabine in the lapatinib expanded access programme, including efficacy in brain metastases-the UK experience. Br J Cancer 2010; 102: 995-1002.

41. Cameron D, Casey M, Press M et al. A phase III randomized comparison of lapatinib plus capecitabine versus capecitabine alone in women with advanced breast cancer that has progressed on trastuzumab: updated efficacy and biomarker analyses. Breast Cancer Research and Treatment 2008; 112: 533-543. 
42. Lin NU, Carey LA, Liu MC et al. Phase II trial of lapatinib for brain metastases in patients with human epidermal growth factor receptor 2-positive breast cancer. Journal of Clinical Oncology 2008; 26: 1993-1999.

43. Lin NU, Dieras V, Paul D et al. Multicenter phase II study of lapatinib in patients with brain metastases from HER2-positive breast cancer. Clin Cancer Res 2009; 15: 1452-1459.

44. Awada A, Colomer R, Inoue K et al. Neratinib Plus Paclitaxel vs Trastuzumab Plus Paclitaxel in Previously Untreated Metastatic ERBB2-Positive Breast Cancer: The NEfERT-T Randomized Clinical Trial. JAMA Oncol 2016; 2: 1557-1564.

45. Krop IE, Lin NU, Blackwell $K$ et al. Trastuzumab emtansine (T-DM1) versus lapatinib plus capecitabine in patients with HER2-positive metastatic breast cancer and central nervous system metastases: a retrospective, exploratory analysis in EMILIA. Ann Oncol 2015; 26: 113-119.

\section{Tables}




\begin{tabular}{|c|c|c|c|}
\hline Parameter & $\begin{array}{c}\mathrm{LX} \\
(N=255)\end{array}$ & $\begin{array}{c}\text { TBP } \\
(N=299)\end{array}$ & $P$ value \\
\hline \multicolumn{4}{|l|}{ Age(year) } \\
\hline$<50$ & $137(53.7 \%)$ & $161(53.8 \%)$ & 0.977 \\
\hline$\geq 50$ & $59(23.1 \%)$ & $95(31.8 \%)$ & 0.024 \\
\hline Unknown & $59(23.1 \%)$ & $43(14.4 \%)$ & 0.008 \\
\hline \multicolumn{4}{|l|}{ Menopausal status } \\
\hline Premenopausal & $40(15.7 \%)$ & $68(20.7 \%)$ & 0.126 \\
\hline Postmenopausal & $182(71.4 \%)$ & $204(68.2 \%)$ & 0.422 \\
\hline Unknown & $33(12.9 \%)$ & $27(9 \%)$ & 0.14 \\
\hline \multicolumn{4}{|l|}{ HR Status } \\
\hline Negative & $136(53.3 \%)$ & $145(48.5 \%)$ & 0.256 \\
\hline Positive & $92(36.1 \%)$ & $139(46.5 \%)$ & 0.013 \\
\hline Unknown & $27(10.6 \%)$ & $15(5 \%)$ & 0.014 \\
\hline Stage $\square$ at initial diagnosis & $32(12.5 \%)$ & $55(18.4 \%)$ & 0.059 \\
\hline \multicolumn{4}{|l|}{ Number of metastatic sites } \\
\hline$<3$ & $178(69.8 \%)$ & $190(63.5 \%)$ & 0.12 \\
\hline$\geq 3$ & $77(30.2 \%)$ & $109(36.5 \%)$ & \\
\hline \multicolumn{4}{|l|}{ Metastases } \\
\hline Lung & $123(48.2 \%)$ & $162(54.2 \%)$ & 0.163 \\
\hline Liver & $109(42.7 \%)$ & $143(47.8 \%)$ & 0.213 \\
\hline Bone & $62(24.3 \%)$ & $86(28.8 \%)$ & 0.238 \\
\hline Brain & $24(9.4 \%)$ & $34(11.4 \%)$ & 0.453 \\
\hline Other & $131(51.4 \%)$ & $150(50.2 \%)$ & 0.78 \\
\hline \multicolumn{4}{|l|}{ Resistance } \\
\hline Primary & $96(37.6 \%)$ & $109(38.1 \%)$ & 0.772 \\
\hline Secondary & $159(62.4 \%)$ & $190(61.9 \%)$ & \\
\hline \multicolumn{4}{|l|}{ Treatment line } \\
\hline 1 & $37(14.5 \%)$ & $43(14.4 \%)$ & 0.966 \\
\hline 2 & $94(36.9 \%)$ & $164(54.8 \%)$ & $\square 0.001$ \\
\hline 3 & $124(48.6 \%)$ & $92(30.8 \%)$ & 0.001 \\
\hline \multicolumn{4}{|l|}{ Previous therapy } \\
\hline \multicolumn{4}{|l|}{ Hormonal } \\
\hline Adjuvant & $76(29.8 \%)$ & $96(32.1 \%)$ & 0.559 \\
\hline Metastatic & $60(23.5 \%)$ & $91(30.4 \%)$ & 0.069 \\
\hline \multicolumn{4}{|l|}{ Radiotherapy } \\
\hline Adjuvant & $86(33.7 \%)$ & $104(34.8 \%$ & 0.794 \\
\hline Metastatic & $44(17.3 \%)$ & $54(18.1 \%)$ & 0.804 \\
\hline \multicolumn{4}{|l|}{ Previous trastuzumab failure } \\
\hline Adjuvant & $37(14.5 \%)$ & $43(14.4 \%)$ & 0.966 \\
\hline Metastatic & $218(85.5 \%)$ & $256(85.6 \%)$ & \\
\hline \multicolumn{4}{|c|}{ Previous trastuzumab treatment } \\
\hline Adjuvant & $78(30.6 \%)$ & $67(22.4 \%)$ & 0.029 \\
\hline Advanced disease only & $177(69.4 \%)$ & $232(77.6 \%)$ & \\
\hline
\end{tabular}




\begin{tabular}{lc}
\hline Table2. chemotherapy combined with trastuzumab \\
\hline Taxane & Patients $(N=299)$ \\
Vinorelbine & $146(48.8 \%)$ \\
\hline Gemcitabine & $33(11 \%)$ \\
\hline Cisplatin & $75(25.1 \%)$ \\
Pemetrexed & $60(20.1 \%)$ \\
Carboplatin & $8(2.7 \%)$ \\
Capecitabine & $6(2 \%)$ \\
\hline
\end{tabular}

\begin{tabular}{|c|c|c|}
\hline ients & $\begin{array}{c}\mathrm{LX} \\
(N=24)\end{array}$ & $\begin{array}{c}\text { TBP } \\
(N=34)\end{array}$ \\
\hline \multicolumn{3}{|l|}{ nber of brain metastatic sites } \\
\hline$<3$ & \multicolumn{2}{|c|}{$21(87.5 \%) 30(88.2 \%)$} \\
\hline$\geq 3$ & $3(12.5 \%)$ & $4(11.8 \%)$ \\
\hline \multicolumn{3}{|l|}{ al treatment } \\
\hline liotherapy (WBRT and/or SRS) & \multicolumn{2}{|c|}{$19(79.2 \%) 28(82.4 \%)$} \\
\hline \multicolumn{3}{|c|}{ irosurgery with WBRT and/or SRS 5(20.8\%) $6(17.6 \%)$} \\
\hline
\end{tabular}

\begin{tabular}{cccc}
\hline Table 4. Central nervous system metastases progression rate & \\
\hline & TBP & LX & $P$ \\
& $(N=248)$ & $(N=203)$ & \\
\hline CNS as new sites of progression & 11 & 6 & \\
\hline Progression of CNS metastases at baseline & 20 & 6 & \\
\hline CNS progression rate & $12.5 \%$ & $5.9 \%$ & 0.018 \\
\hline
\end{tabular}




\begin{tabular}{|c|c|c|c|c|}
\hline & & & & \\
\hline & \multicolumn{2}{|c|}{$(N=236)$} & \multicolumn{2}{|c|}{$(N=296)$} \\
\hline & grade1-2 & grade3-4 & grade1-2 & grade3-4 \\
\hline ia & $24(10.2 \%)$ & $5(2.1 \%)$ & $87(29.4 \%)$ & $19(6.4 \%)$ \\
\hline atropenia & $4(1.7 \%)$ & $0(0.0 \%)$ & $20(6.8 \%)$ & $4(1.4 \%)$ \\
\hline \multirow[t]{2}{*}{ topenia } & $12(5.1 \%)$ & $1(0.4 \%)$ & $25(8.4 \%)$ & $3(1 \%)$ \\
\hline & $4(1.7 \%)$ & $0(0.0 \%)$ & $40(13.5 \%)$ & $0(0.0 \%)$ \\
\hline \multirow[t]{2}{*}{ miting } & $60(25.4 \%)$ & $0(0.0 \%)$ & $56(18.9 \%)$ & $8(2.7 \%)$ \\
\hline & $92(39.0 \%)$ & $12(5.1 \%)$ & $15(5.1 \%)$ & $0(0.0 \%)$ \\
\hline xicity & $0(0.0 \%)$ & $0(0.0 \%)$ & $2(0.7 \%)$ & $0(0.0 \%)$ \\
\hline ythema & $45(19.1 \%)$ & $0(0.0 \%)$ & $13(4.4 \%)$ & $0(0.0 \%)$ \\
\hline ncreased & $28(11.9 \%)$ & $0(0.0 \%)$ & $32(10.8 \%)$ & $27(9.1 \%)$ \\
\hline syndrome & $56(23.7 \%)$ & $24(10.2 \%)$ & $7(2.4 \%)$ & $0(0.0 \%)$ \\
\hline
\end{tabular}

Figures 

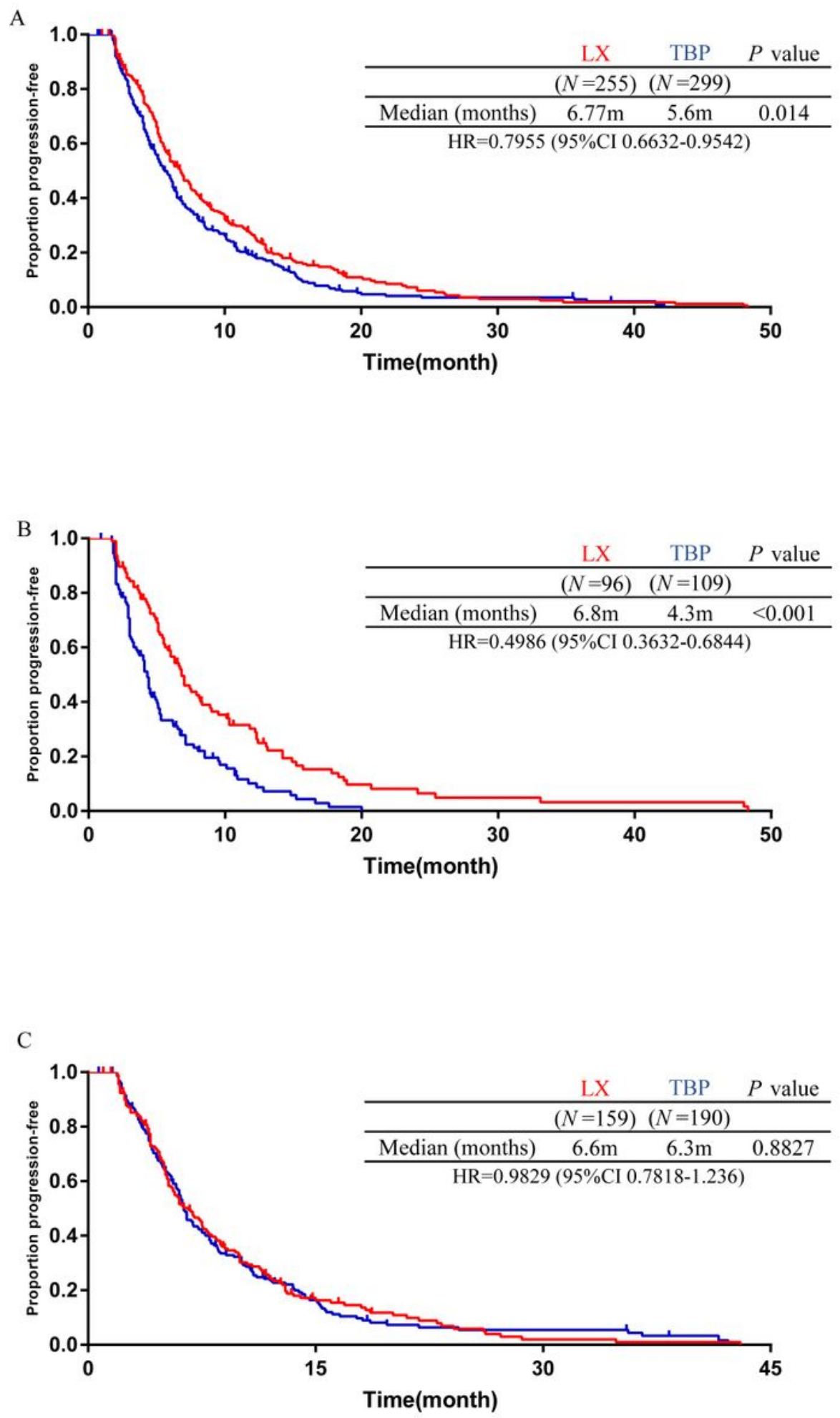

\section{Figure 1}

Kaplan-Meier analysis of progression-free survival (A) PFS in all patients (B) PFS in the primary resistant population (C) PFS in the secondary resistant population. $\mathrm{Cl}$, confidence interval; HR, hazard ratio; $\mathrm{m}$, months; PFS, progression-free survival; LX, lapatinib plus capecitabine; TBP, trastuzumab beyond progression; 


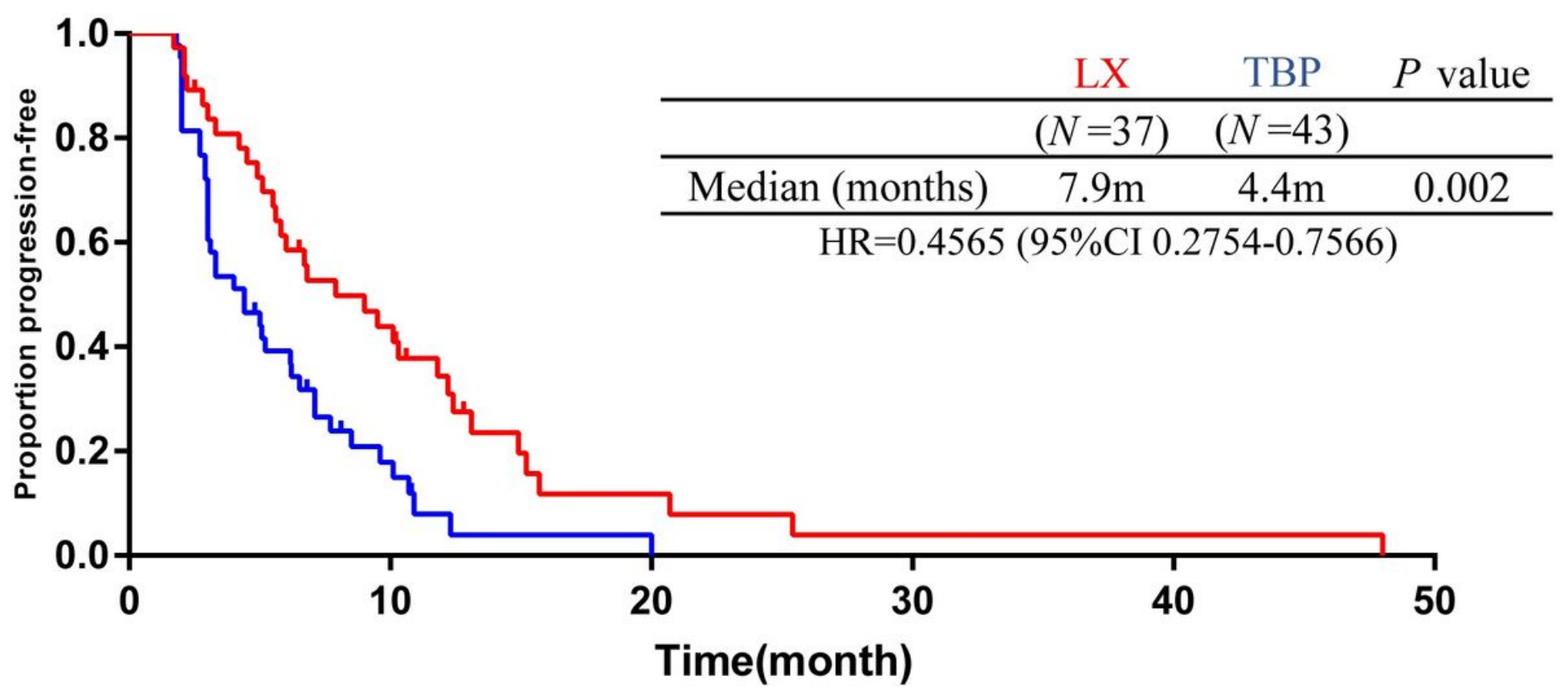

Figure 2

Kaplan-Meier analysis of progression-free survival in first line treatment population 

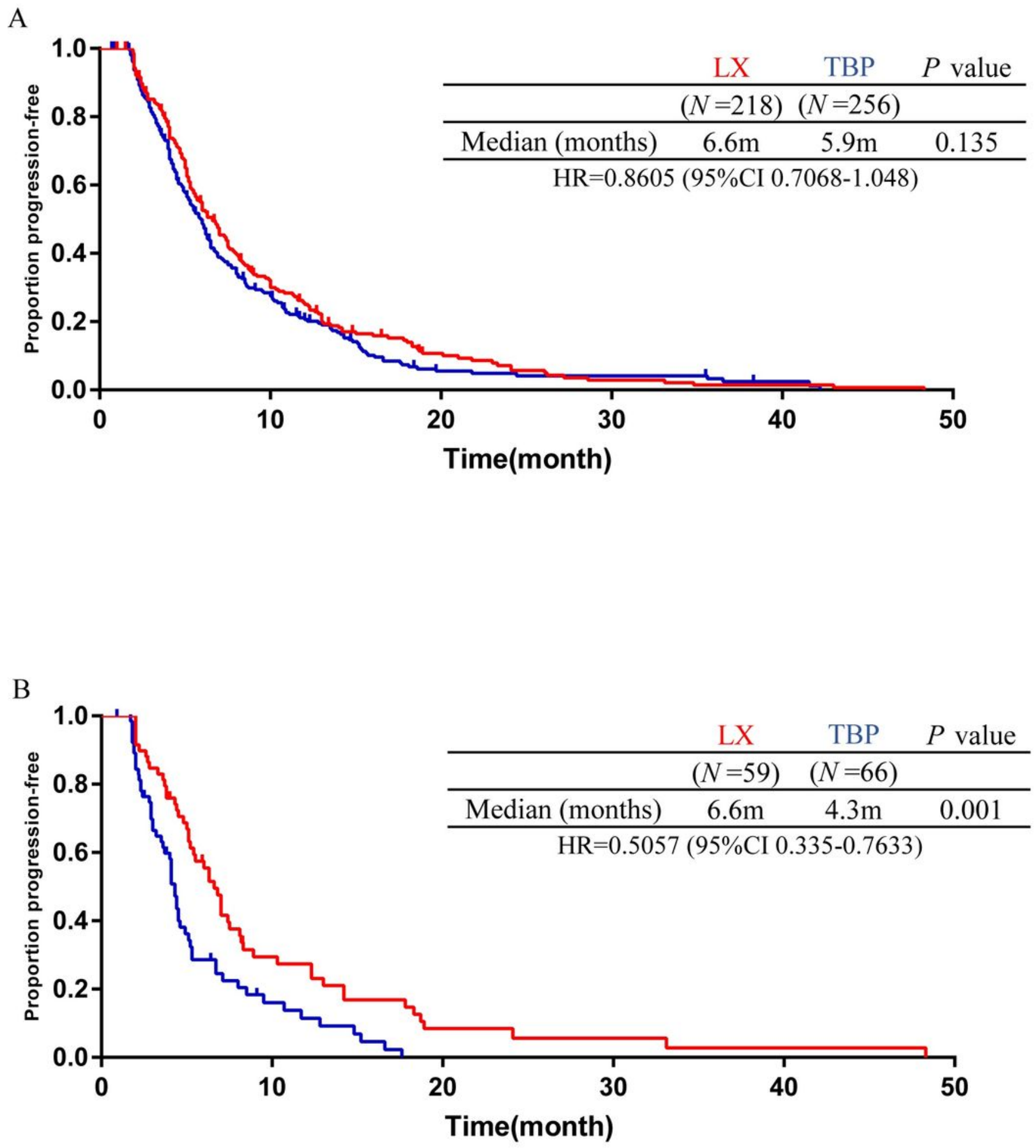

Figure 3

Kaplan-Meier analysis of progression-free survival in second and third line treatment population (A) PFS in all patients (B) PFS in the primary resistant population 
Other metastases(yes vs no)

Lung metastases(yes vs no)

Liver metastases(yes vs no)

Bone metastases(yes vs no)

Brain metastases(yes vs no)

Secondary vs primary resistance

Third line treatment

Second line treatment

First line treatment*

HR(negative vs positive)

Age $\leq 50$ vs $>50$

TBP vs LX

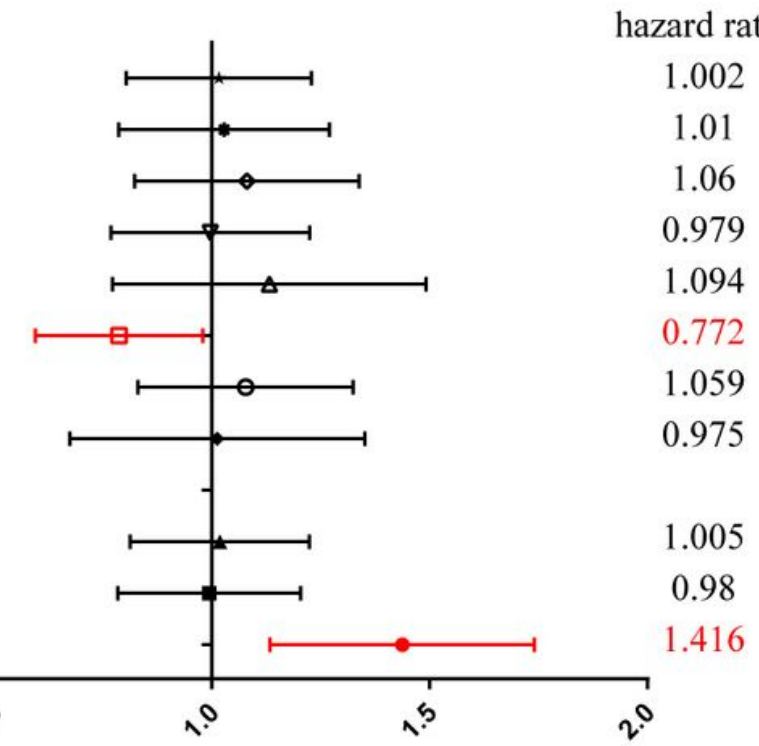

\section{Figure 4}

Multivariate analysis for progression-free survival Derived from the Cox regression model. HR hormone receptors status ; *Reference group
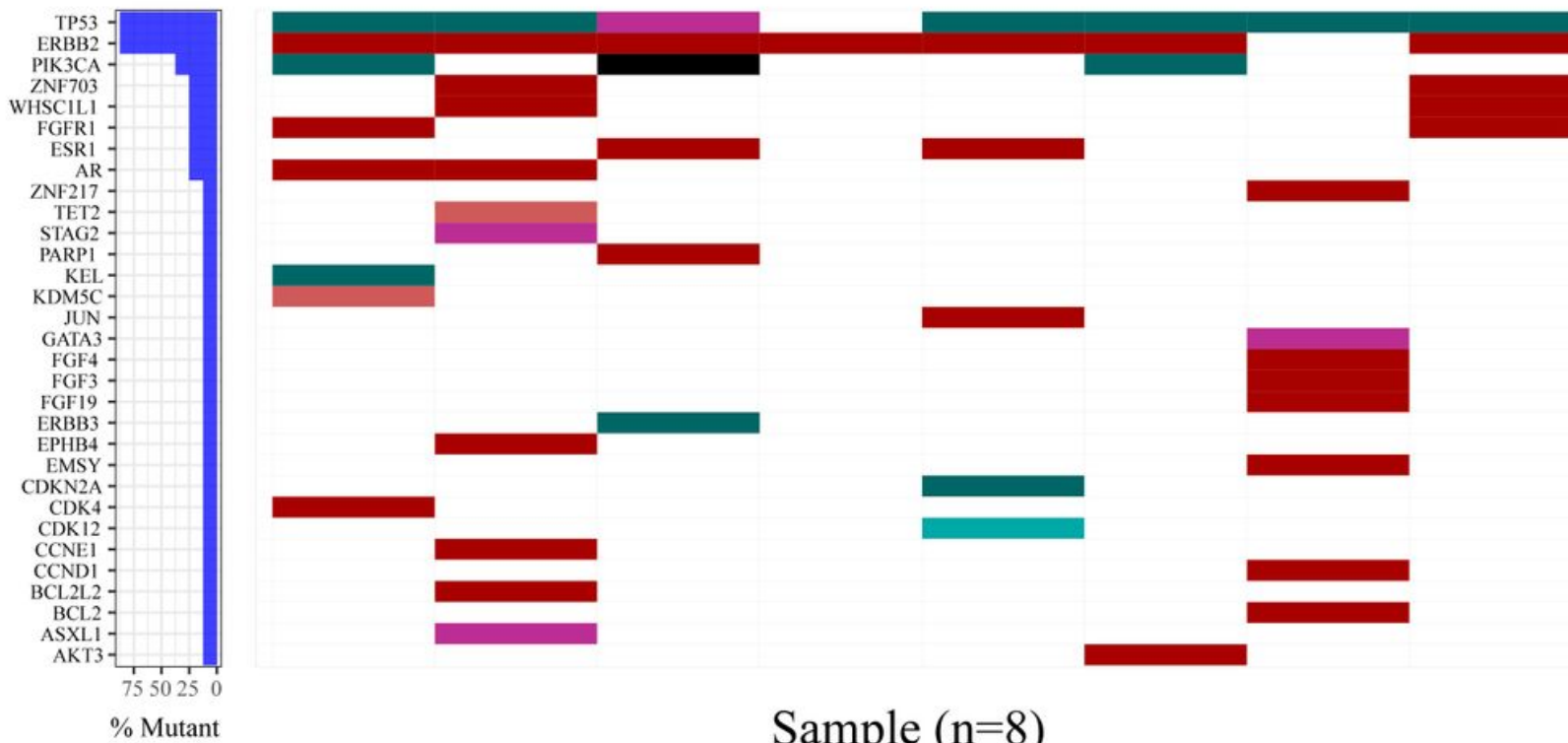

Mutation Type

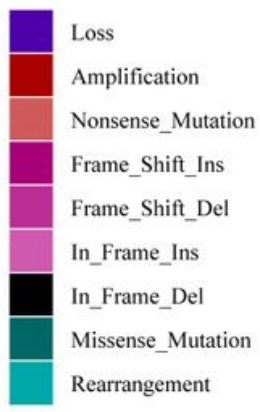

Sample $(\mathrm{n}=8)$

Figure 5

Comprehensive annotation of genomic alterations in this study. 


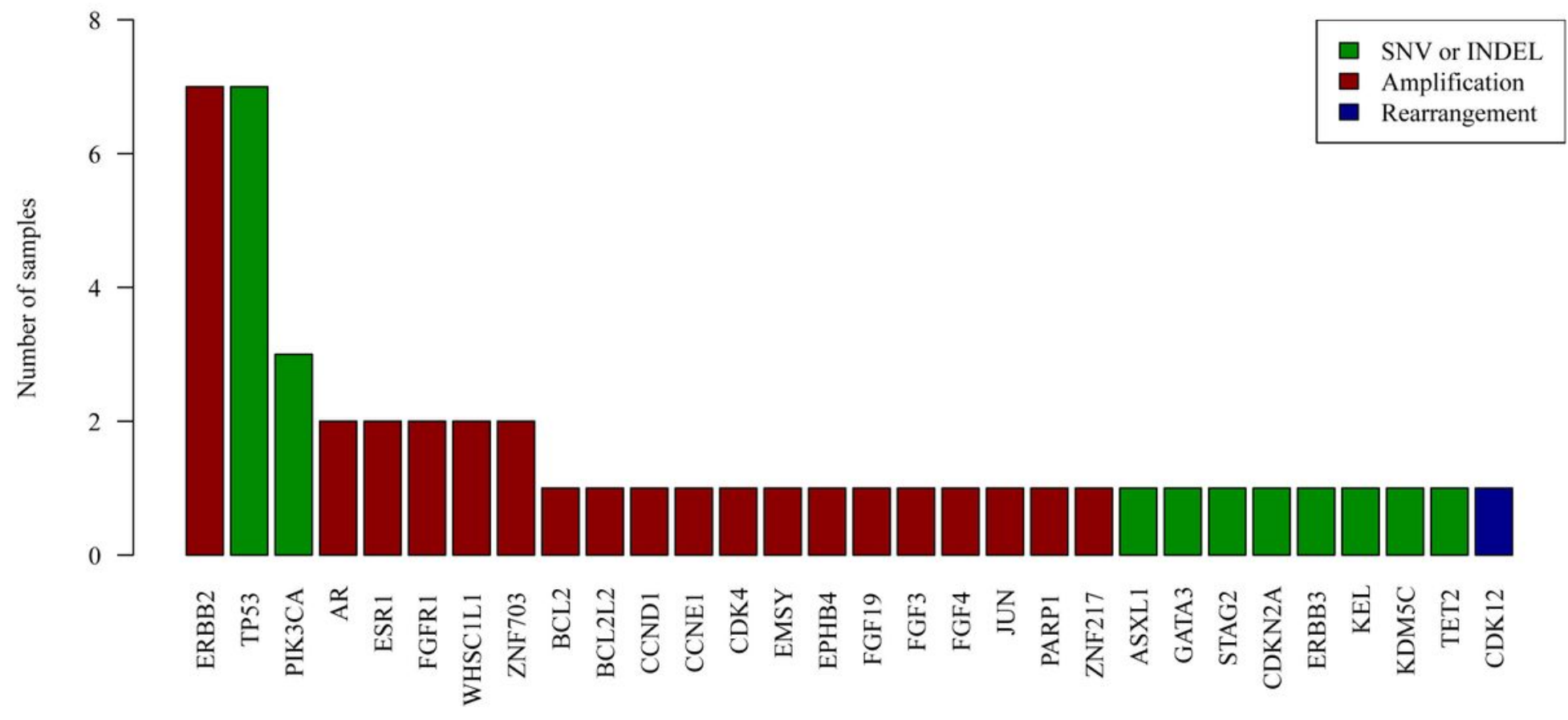

Figure 6

Distribution of alterations observed in eight patients 

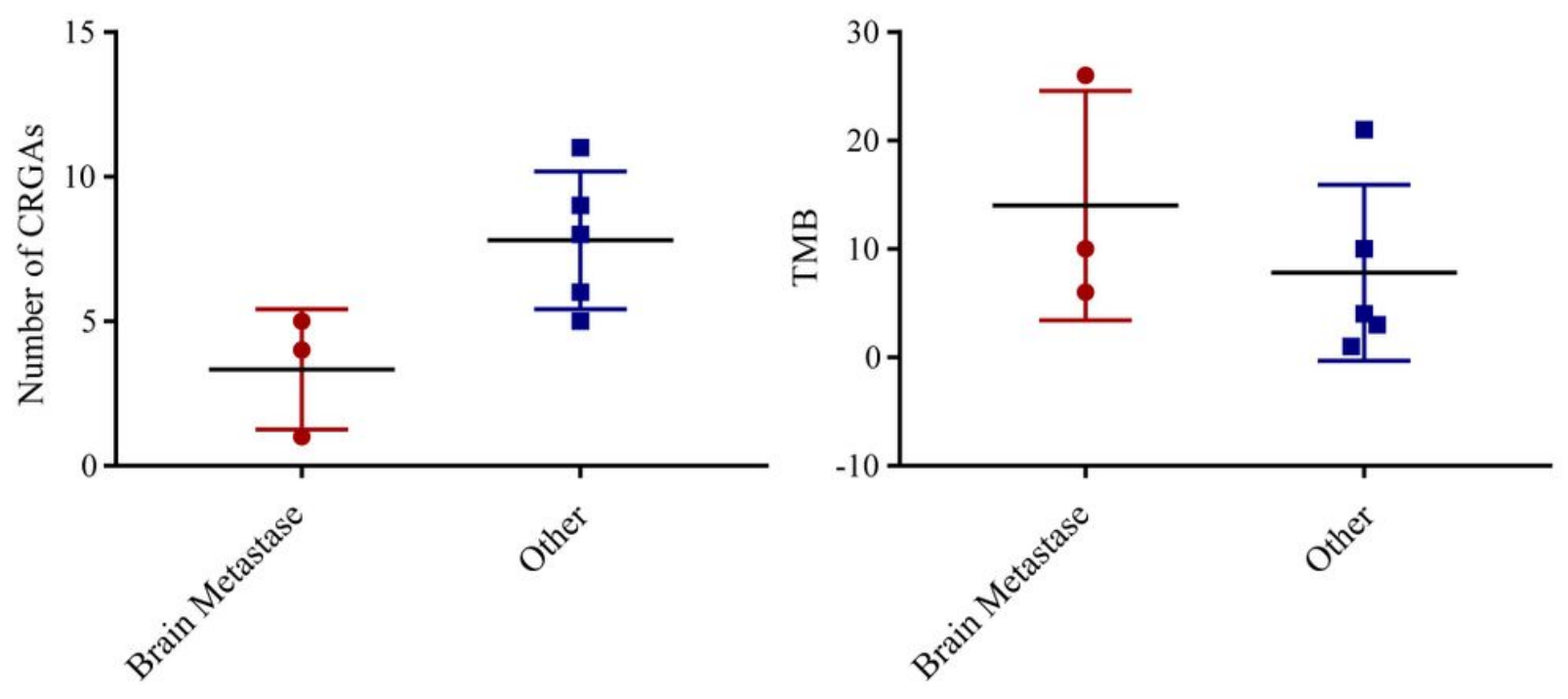

Figure 7

The number of tumor mutation burdens and CRGAs in patients with Extracranial metastases compared with brain metastases 

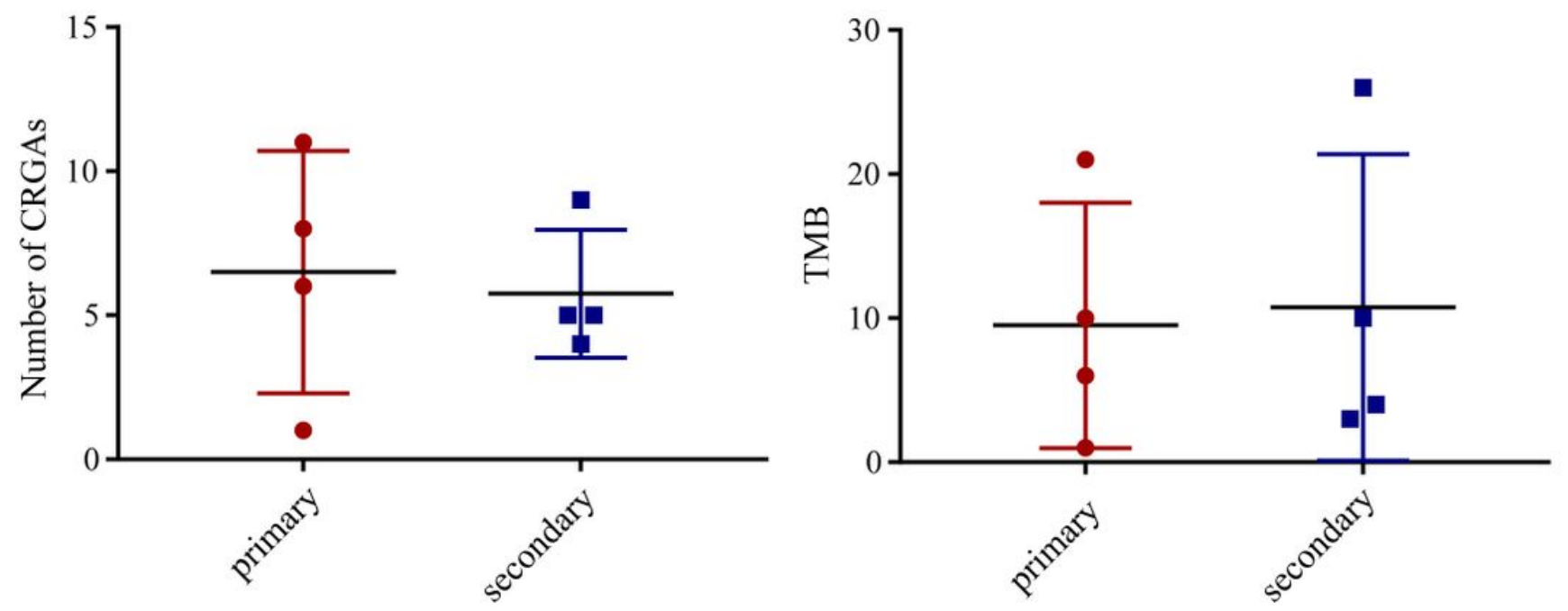

Figure 8

The number of tumor mutation burdens and CRGAs in primary and secondary resistant patients

\section{Supplementary Files}

This is a list of supplementary files associated with this preprint. Click to download.

- S2.doc 